\title{
ABUNDANCIA Y DISTRIBUCIÓN DE LAS ESPECIES ÍCTICAS (OSTEICHTHYES) DEL RÍO SAN FRANCISCO-COSQUÍN, CÓRDOBA, ARGENTINA
}

\author{
Andrea Cecilia Hued ${ }^{1}$ \\ María de los Ángeles Bistoni ${ }^{1}$
}

\begin{abstract}
ABUNDANCE AND DISTRIBUTION OF FISH SPECIES (OSTEICHTHYES) FROM SAN FRANCISCO - COSQUÍN RIVER IN CÓRDOBA, ARGENTINA. It was made the characterization of the San Francisco-Cosquín Mountain River fish community, Córdoba, Argentina. Samples of fish were taken from selected sites from November 1998 to November 1999. Eleven species were recorded, eight of which are first reports for this river. Relative abundance for each species, species richness, diversity and dominance were estimated. Bryconamericus iheringi (Boulenger, 1887) y Jenynsia multidentata (Jenyns, 1842) were the most abundant.
\end{abstract}

KEYWORDS. Fish, freshwater, community attributes.

\section{INTRODUCCIÓN}

El río San Francisco-Cosquín (Córdoba, Argentina) se caracteriza por la presencia de asentamientos humanos y centros de importancia turística sobre sus márgenes, lo que incrementa el riesgo de contaminación y deterioro de los hábitats acuáticos. Por este motivo es prioritario investigar la composición específica de sus aguas, aún pobremente conocida. El único estudio referido a la fauna de peces del curso mencionado fue realizado por MenNi et al. (1984), quienes confeccionaron una lista de peces de los ríos de las áreas serranas de las provincias de Córdoba y San Luis (Argentina), basada en colectas, en general, ocasionales.

El objetivo fue realizar un relevamiento sistemático de la ictiofauna del río San Francisco-Cosquín y desarrollar algunas consideraciones sobre la abundancia y distribución de las especies a lo largo del curso.

\section{MATERIAL Y MÉTODOS}

Perteneciente a la cuenca endorreica del Río Primero, también conocida como Suquía, y ubicado en el Departamento Punilla, provincia de Córdoba, Argentina, el río San Francisco-Cosquín nace en el cordón montañoso de las Sierras Grandes, corre hacia el sur y conjuntamente con el río San Antonio vuelca sus aguas al Lago San Roque, dique de origen artificial (VÁZQUEZ et al., 1979). En sus primeros tramos el río es de régimen rápido y torrencial, con mayor pendiente y lecho de rocas y cantos rodados. Río abajo la pendiente es menor y disminuye la granulometría del sustrato, siendo los fondos más arenosos. De acuerdo a la accesibilidad al curso se determinaron cuatro sitios de muestreo: 1, Huerta Grande; 2, Valle Hermoso; 3, Cosquín y 4, Villa Bustos (fig. 1). En cada sitio se realizaron dos muestreos en el período hidrológico seco (Abril-Septiembre) y dos en el período de caudales altos (Octubre-Marzo), contabilizándose en total 16 viajes de campaña, entre Noviembre de 1998 y Noviembre de 1999.

Se utilizó pesca eléctrica para la captura de los peces. Este método es el más apropiado para cursos serranos que poseen aguas cristalinas y fondo rocoso (VINCENT, 1971), presentando valores de conductividad adecuados para su correcto funcionamiento $(0,13$ a $0,61 \mathrm{mS} / \mathrm{cm})$. Las colectas fueron realizadas sobre un tramo de 150 metros (KARR,

1. Cátedra de Diversidad Animal II. Facultad de Ciencias Exactas, Físicas y Naturales. Universidad Nacional de Córdoba. Av. Vélez Sársfield 299, 5000 - Córdoba, Argentina. (achued@com.uncor.edu) 
1981), donde fueron muestreados los distintos hábitats del río (zonas de corriente, de aguas quietas, lugares con vegetación acuática o costera). Los individuos atraídos por el electroshock fueron recolectados con redes de mano.

Las especies capturadas fueron identificadas en campaña y laboratorio mediante claves sistemáticas (EigenMann, 1915; Eigenmann, 1927; Ringuelet et al., 1967; GeRY, 1977; Gery et al., 1987). Los ejemplares fueron fijados en formol $10 \%$, luego conservados en alcohol $80 \%$ y se encuentran depositados en la Cátedra de Diversidad Animal II (Vertebrados) (CDAII P9) a cargo de la Dra. María de los Ángeles Bistoni, Facultad de Ciencias Exactas, Físicas y Naturales, Universidad Nacional de Córdoba.

Todos los peces fueron contados y se estimó la abundancia de cada especie, según la captura por unidad de esfuerzo (CPUE), para cada sitio de muestreo y período hidrológico, utilizando: $n_{i}=c_{i} / t$, en donde $n_{i}$ es la abundancia relativa de la especie $\mathrm{i}, \mathrm{c}_{\mathrm{i}}$ la cantidad de individuos recolectados de esa especie y $\mathrm{t}$ los segundos de la actividad del electroshock (VIDELA \& BISTONI, 1999).

Para cada sitio de muestreo y para cada período hidrológico, se estimaron los siguientes atributos de la comunidad: abundancia por especie, riqueza de especies, dominancia a través del índice de Berger-Parker (MAGURRAN, 1988) y diversidad, por medio del índice de Shannon-Wiener (WaShington, 1984). Las diferencias de valores de este último atributo para cada punto de muestreo fueron evaluados mediante el test-t (SOKAL \& ROHLF, 1979), con un nivel de significancia del $5 \%$.

\section{RESULTADOS Y DISCUSIÓN}

Dentro del esquema ictiogeográfico propuesto por RINGUELET (1975), el sistema endorreico del río Primero se encuentra comprendido en la provincia Parano-platense del dominio Paranaense. Su ubicación, vecina a la provincia Subandina, del dominio Andino, hace que este río fluya en una zona ecotonal notable entre estos dos dominios geográficos. Tal situación motiva la presencia de formas pertenecientes a ambas provincias ictiogeográficas.

En el curso estudiado predominaron notablemente las formas paranaenses $(81,8 \%)$, a excepción de Rineloricaria catamarcensis (Berg, 1985), representante de la fauna andina, aunque en la actualidad se discute tal condición (ARratia et al., 1983), y de la

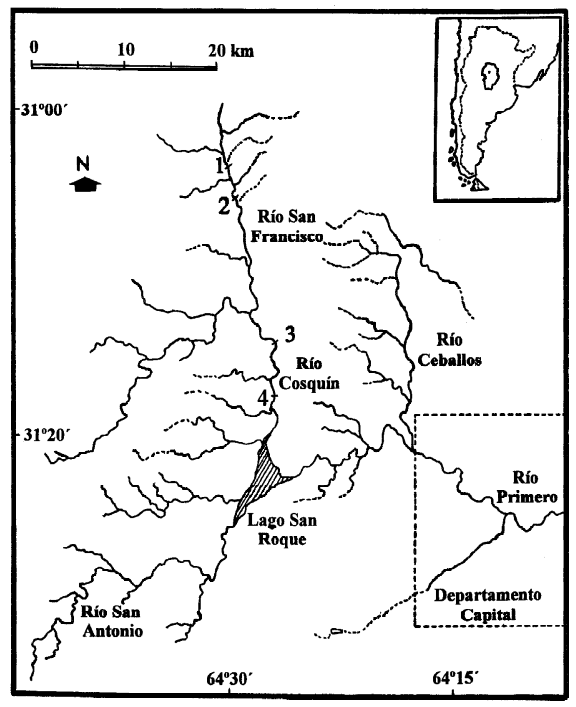

Fig. 1. Localización geográfica del área de estudio y sitios de muestreo: 1, Huerta Grande; 2, Valle Hermoso; 3 , Cosquín; 4, Villa Bustos. 
mojarra Astyanax eigenmanniorum (Cope, 1894), representante de la fauna transicional, común en aguas serranas y ausente del Paraná (Ringuelet, 1975).

Los relevamientos efectuados permitieron capturar 1390 individuos. Estos ejemplares están representados por 11 especies, agrupadas en 5 órdenes y 6 familias (tab. I), que corresponde al $23,9 \%$ de la fauna citada para la provincia de Córdoba por Haro \& Bistoni (1996). Fueron capturadas 8 especies no listadas en el estudio de MenNi et al. (1984): Oligosarcus jenynsia, Pimelodella laticeps, Rhamdia cf. quelen, Rineloricaria catamarcensis, Hypostomus cordovae, Jenynsia multidentata, Cnesterodon decenmaculatus y Synbranchus marmoratus.

Bryconamericus iheringi fue la especie más abundante en los tramos superiores de la cuenca y Jenynsia multidentata en los inferiores (tab. I). La riqueza específica presentó poca variación a lo largo del río (tab. II). La diversidad varió significativamente entre los sitios 1 (Huerta Grande) y 2 (Valle Hermoso) ( $\mathrm{t}=2,51)$ y entre los puntos 3 (Cosquín) y 4 (Villa Bustos) $(\mathrm{t}=3,45)(\mathrm{p}<0,05)$. Se observa que los valores son menores en los puntos 2 y 4. Antes de llegar a estas localidades el río atraviesa importantes centros urbanos como La Falda y Cosquín. Estas ciudades que no cuentan con servicios cloacales, vierten los desechos al lecho del curso por lo que las aguas presentan una menor calidad, siendo menos aptas para el desarrollo de comunidades acuáticas más diversas.

La especie dominante en ambas estaciones hidrológicas fue Bryconamericus iheringi (tab. III). Esta especie así como también Rineloricaria catamarcensis e Hypostomus cordovae disminuyeron su abundancia en el período lluvioso. Lo contrario

Tabla I. Abundancia relativa de las especies ícticas en cada sitio de muestreo y abundancia relativa total para el río San Francisco-Cosquín, Argentina, en Noviembre 1998/1999 (1, Huerta Grande; 2, Valle Hermoso; 3, Cosquín; 4, Villa Bustos).

\begin{tabular}{|c|c|c|c|c|c|}
\hline \multirow[b]{2}{*}{ ESPECIES } & \multicolumn{4}{|c|}{$\begin{array}{c}\text { Abundancia Relativa de las especies en cada } \\
\text { sitio de muestreo }\end{array}$} & \multirow{2}{*}{$\begin{array}{l}\text { Abundancia } \\
\text { relativa } \\
\text { total }\end{array}$} \\
\hline & 1 & 2 & 3 & 4 & \\
\hline \multicolumn{6}{|l|}{ CHARACIFORMES } \\
\hline \multicolumn{6}{|l|}{ Characidae } \\
\hline Oligosarcus jenynsi (Günther, 1864) & 0,14 & & 0,86 & & 1,00 \\
\hline Astyanax eigenmanniorum (Cope, 1894) & 4,12 & & 1,18 & 0,63 & 5,93 \\
\hline Bryconamericus iheringi (Boulenger, 1887) & 34,41 & 59,80 & 1,81 & 0,73 & 96,75 \\
\hline Cheirodon interruptus (Jenyns, 1842) & 2,51 & & 0,37 & 3,32 & 6,20 \\
\hline \multicolumn{6}{|l|}{ SILURIFORMES } \\
\hline \multicolumn{6}{|l|}{ Pimelodidae } \\
\hline Pimelodella laticeps Eigenmann, 1917 & 1,07 & & 1,59 & 1,06 & 3,72 \\
\hline Rhamdia cf. quelen (Quoy \& Gaimard, 1824) & & 0,11 & & 0,37 & 0,48 \\
\hline \multicolumn{6}{|l|}{ Loricariidae } \\
\hline Rineloricaria catamarcensis (Berg, 1895) & 1,89 & 1,15 & 1,92 & 1,13 & 6,09 \\
\hline Hypostomus cordovae (Günther, 1880) & 2,95 & 1,73 & 1,68 & 1,23 & 7,59 \\
\hline \multicolumn{6}{|l|}{ ATHERINIFORMES } \\
\hline \multicolumn{6}{|l|}{ Jenynsiidae } \\
\hline Jenynsia multidentata (Jenyns, 1842) & 6,39 & 6,24 & 1,30 & 34,85 & 48,78 \\
\hline Poeciliidae & & & & & \\
\hline Cnesterodon decenmaculatus (Jenyns, 1842) & 3,73 & 23,58 & & 0,81 & 28,12 \\
\hline \multicolumn{6}{|l|}{ SYNBRANCHIFORMES } \\
\hline Synbranchidae & & & & & \\
\hline Synbranchus marmoratus Bloch, 1795 & 0,34 & 0,25 & 0,23 & 1,05 & 1,62 \\
\hline
\end{tabular}


Tabla II. Variación de los atributos de la comunidad íctica a lo largo del río San Francisco-Cosquín en Noviembre 1998/1999 (1, Huerta Grande; 2, Valle Hermoso; 3, Cosquín; 4, Villa Bustos).

\begin{tabular}{lrrrr}
\hline Atributos de la comunidad íctica & \multicolumn{4}{c}{ Sitios de muestreo } \\
\cline { 2 - 5 } & \multicolumn{1}{c}{1} & \multicolumn{1}{c}{3} & 4 \\
\hline Abundancia relativa & 57,55 & 92,86 & 10,94 & 45,18 \\
Riqueza & 10 & 7 & 9 & 10 \\
Diversidad & 1,44 & 0,97 & 2,06 & 0,99 \\
Dominancia & 0,59 & 0,64 & 0,18 & 0,77 \\
\hline
\end{tabular}

Tabla III. Variación estacional de los porcentajes de abundancia relativa de cada especie y de los atributos de la comunidad íctica del río San Francisco-Cosquín en Noviembre 1998/1999.

\begin{tabular}{lcc}
\hline & $\begin{array}{c}\text { Estación } \\
\text { Seca } \\
\%\end{array}$ & $\begin{array}{c}\text { Estación } \\
\text { Lluviosa } \\
\%\end{array}$ \\
\hline Especies & 0,65 & 0,35 \\
Oligosarcus jenynsi & 3,57 & 3,46 \\
Astyanax eigenmanniorum & 50,87 & 38,98 \\
Bryconamericus iheringi & 2,38 & 3,07 \\
Cheirodon interruptus & 1,95 & 2,20 \\
Pimelodella laticeps & 0,00 & 0,39 \\
Rhamdia cf. quelen & 4,32 & 1,65 \\
Rineloricaria catamarcensis & 14,07 & 4,09 \\
Hypostomus cordovae & 19,48 & 24,36 \\
Jenynsia multidentata & 1,84 & 20,43 \\
Cnesterodon decenmaculatus & 0,87 & 1,02 \\
Synbranchus marmoratus & & \\
\hline
\end{tabular}

ocurrió con los orilleros Jenynsia multidentata y Cnesterodon decenmaculatus quienes incrementaron su abundancia en la estación de caudales altos, lo cual estaría relacionado con los hábitos reproductivos de estas especies, ya que en esta época se recolectaron numerosos juveniles de ambas especies.

Agradecimientos. A Nancy Hued, Martín Caruso y Sebastián Dardanelli (todos de la Universidad Nacional de Córdoba), por la colaboración en el trabajo de campo. Para la realización de este estudio se contó con subsidio otorgado por el Consejo Nacional de Investigaciones Científicas y Técnicas (CONICET), Argentina.

\section{REFERENCIAS BIBLIOGRÁFICAS}

Arratia, G.; Peñafort, M. B. \& Menu-Marque, S. 1983. Peces de la región sureste de los Andes y sus probables relaciones biogeográficas actuales. Deserta, Mendoza, 7:48-107.

Eigenmann, C. H. 1915. The Cheirodontinae, a subfamily of minute characid fishes of South America. Mem. Carneg. Mus., Pittsburgh, 7(1):1-99.

1927. The American Characidae. Mem. Mus. Comp. Zool. Harv., Cambridge, Mass., 43(5):429-558.

GERY, J. 1977. Characoids of the world. Neptune, T.F.H. Publ. 672p.

Gery, J.; Mahnert, V. \& Louhy, D. 1987. Poissons Characoides non Characidae du Paraguay (Pisces, Ostariophysi). Revue suisse Zool., Gèneve, 94:357-464.

Haro, J. G. \& Bistoni, M. A. 1996. Ictiofauna de la Provincia de Córdoba. In: Di TAda, I. E. \& Bucher, E. H. eds. Biodiversidad de la Provincia de Córdoba. Fauna. Córdoba, v. 1, p. 169-190.

KarR, J. R. 1981. Assessment of biotic integrity using fish communities. Fisheries, Maryland, 6:21-27.

Magurran, A. 1988. Ecological Diversity and its Measurement. London, Chapman \& Hall. 179p.

Menni, R. C.; LóPez, H. L. et al. 1984. Ictiología de las áreas serranas de Córdoba y San Luis (Argentina). Biología Acuática, La Plata, 5:1-63.

Ringuelet, R. A. 1975. Zoogeografía y ecología de los peces de aguas continentales de la Argentina y consideraciones sobre las áreas ictiológicas de América del Sur. Ecosur, Corrientes, 2:1-151.

Ringuelet, R. A.; Arámburu, R. H. \& Arámburu, A. A. de. 1967. Los peces argentinos de agua dulce. La Plata, Comisión de Investigación Científica. 602p.

SoKal, R. R. \& RoHLF, F. J. 1979. Biometría: Principios y métodos estadísticos en investigación biológica. Madrid, H. Blume. 832p.

Vázquez, J. B.; López Robles, A. et al. 1979. Aguas. In: Vázquez, J. B.; Miatello, R. A. \& Roque, M. E. Geografía Física de la Provincia de Córdoba. Buenos Aires, Boldt, p. 139-211.

Vincent, R. 1971. River electrofishing and fish population estimates. The Progres. Fish-Cult., Maryland, 33(3):653-694.

Washington, H. G. 1984. Diversity, biotic and similarity indices. Water Res., Oxford, 18(6):653-694.

Recebido em 08.03.2000; aceito em 29.03.2001. 